\title{
JLC. Authors and/or Referees (THEMA 1, 2 ; VARIA 1, 2, 3).
}

JLC. Authors (THEMA 1, 2 ; VARIA 1, 2, 3)

\begin{tabular}{|c|c|c|}
\hline Alexandre & Nélia & University of Lisbon, Portugal \\
\hline Avram & Andrei A. & University of Bucharest, Romania \\
\hline Beyer & Klaus & Humboldt University - Berlin, Germany \\
\hline Boutin & Béatrice Akissi & Université de Toulouse-Le Mirail, France \\
\hline Caïtucoli & Claude & Université de Rouen, France \\
\hline Carpitelli & Elisabetta & Université de Grenoble III, France \\
\hline Comrie & Bernard & $\begin{array}{l}\text { Max Planck Institute for Evolutionary } \\
\text { Anthropology, Leipzig, Germany }\end{array}$ \\
\hline Enfield & Nicholas J. & $\begin{array}{l}\text { Max Planck Institute for Psycholinguistics, } \\
\text { Nijmegen, Netherlands }\end{array}$ \\
\hline Estève & Isabelle & Université Stendhal-Grenoble3, France \\
\hline Féral & Carole de & Université de Nice-Sophia Antipolis, France \\
\hline Fleisch & Axel & University of Helsinki, Finland \\
\hline Frajzyngier & Zygmunt & University of Colorado, Boulder, USA \\
\hline Gadet & Françoise & Université de Paris X, France \\
\hline Grant & Anthony & Edge Hill University, England \\
\hline Hagemeijer & Tjerk & University of Lisbon, Portugal \\
\hline Heine & Bernd & University of Cologne, Germany \\
\hline Heinold & Simone & University of Stuttgart, Germany \\
\hline Jarva & Vesa & ELTE University, Budapest, Hungary \\
\hline Jones & Mari C. & University of Cambridge, England \\
\hline Juillard & Caroline & Université Paris V, France \\
\hline Kuteva & Tania & University of Düsseldorf, Germany \\
\hline Lefebvre & Claire & Université du Québec à Montréal, Canada \\
\hline Lipski & John M. & The Pennsylvania State University, USA \\
\hline McConvell & Patrick & AIATSIS, Canberra, Australia \\
\hline Millet & Agnès & Université Stendhal-Grenoble3, France \\
\hline Mithun & Marianne & University of California, Santa Barbara, USA \\
\hline Mondada & Lorenza & Université de Lyon II, France \\
\hline Montgomery-Anderson & Brad & Northeastern State University, Tahlequah, USA \\
\hline Mufwene & Salikoko S. & University of Chicago, USA \\
\hline Myers- Scotton & Carol & Université Marc Bloch, France \\
\hline Newman & Zelda Kahan & City University of New York, USA \\
\hline Nicolaï & Robert & Université de Nice, France \\
\hline Owens & Jonathan & University of Bayreuth, Germany \\
\hline Pakendorf & Brigitte & $\begin{array}{l}\text { Max Planck Institute for Evolutionary } \\
\text { Anthropology, Leipzig, Germany }\end{array}$ \\
\hline Petitjean & Cécile & Université de Provence, France \\
\hline Pietsch & Lukas & University of Hamburg, Germany \\
\hline Ploog & Katja & Université de Franche-Comte, France \\
\hline Py & Bernard & Université de Neuchâtel, Swiss \\
\hline Quint & Nicolas & LLACAN - CNRS, France \\
\hline Ross & Malcom & The Australian National University, Aistralia \\
\hline Samarin & William J. & University of Toronto, Canada \\
\hline
\end{tabular}

Journal of Language Contact - VARIA 3 (2010)

www. jlc-journal.org 


$\begin{array}{lll}\text { Shay } & \text { Erin } & \text { University of Colorado, Boulder, USA } \\ \text { Shin } & \text { Naomi Lapidus } & \text { University of Montana, USA } \\ \text { Silva-Corvalán } & \text { Carmen } & \text { University of Southern California, USA } \\ \text { Sinner } & \text { Carsten } & \text { University of Leipzig, Germany } \\ \text { Smit } & \text { Merlijn de } & \text { Stockholm University, Sweden } \\ \text { Speedy } & \text { Karin } & \text { Macquarie University - Sydney, Australia } \\ \text { Sullivan } & \text { Celeste } & \text { Brown University, Providence, India. } \\ \text { Tabouret-Keller } & \text { Andrée } & \text { Université Université Marc Bloch, , France } \\ \text { Thomason } & \text { Sarah G. } & \text { University of Michigan, USA } \\ \text { Thurgood } & \text { Graham } & \text { California State University, Chico, USA } \\ \text { Tosco } & \text { Mauro } & \text { University of Torino, Italia } \\ \text { Véronique } & \text { Georges D. } & \text { Université de Provence, France } \\ \text { Winford } & \text { Donald } & \text { Ohio State University, USA } \\ \text { Zima } & \text { Petr } & \text { Charles University, Praha, Czech Republic } \\ \text { Zuckermann } & \text { Ghil'ad } & \text { University of Queensland, Australia }\end{array}$

JLC. Referees (THEMA 1, 2 ; VARIA 1, 2, 3).

\begin{tabular}{|c|c|c|}
\hline Aikhenvald & Alexandra & James Cook University, Australia \\
\hline Auer & Peter & University of Freiburg, Germany \\
\hline Caubet & Dominique & INALCO, Paris, France \\
\hline Chaker & Salem & Université d'Aix-en-Provence, France \\
\hline Cheshire & Jenny & Queen Mary, University of London, England \\
\hline Creissels & Denis & Université de Lyon II, France \\
\hline Deuchar & Margaret & Bangor University, United Kingdom \\
\hline Dumestre & Gérard & INALCO, Paris, France \\
\hline Féral & Carole de & Université de Nice-Sophia Antipolis, France \\
\hline Frajzyngier & Zygmunt & University of Colorado, Boulder, USA \\
\hline Gadet & Françoise & Université de Paris X, France \\
\hline Grenoble & Lenore A. & University of Chicago, USA \\
\hline Gupta & Anthea Fraser & University of Leeds, England \\
\hline Guy & Gregory R. & New York University, USA \\
\hline Heath & Jeffrey & University of Michigan, USA \\
\hline Heine & Bernd & University of Cologne, Germany \\
\hline Karangwa & Jean de Dieu & INALCO, Paris, France \\
\hline Kastenholz & Raimund & Johannes Gutenberg University, Mainz, Germany \\
\hline Keiser & Steve Hartman & Marquette University, Wisconsin, USA \\
\hline Lipski & John M. & The Pennsylvania State University, USA \\
\hline Lodge & Anthony & St Andrews University, London, England \\
\hline Martin & Fabienne & Stuttgart University, Germany \\
\hline Meakins & Felicity & University of Manchester England \\
\hline Meinschaefer & Judith & $\begin{array}{l}\text { Julius-Maximilans Würzburg University, } \\
\text { Germany }\end{array}$ \\
\hline Miller & Catherine & IREMAM, France \\
\hline Mithun & Marianne & University of California, Santa Barbara, USA \\
\hline Muyskens & Pieter & Amsterdam University, Netherlands \\
\hline O'Shannessy & Carmel & University of Michigan, USA \\
\hline Otheguy & Ricardo & City University of New York, USA \\
\hline Owens & Jonathan & University of Bayreuth, Germany \\
\hline Pietrandrea & Paola & University of Rome TRE, Italia \\
\hline Ploog & Katja & Université de Franche-Comté, France \\
\hline Salmons & Joseph & University of Wisconsin, USA \\
\hline
\end{tabular}




$\begin{array}{lll}\text { Samarin } & \text { William J. } & \text { University of Toronto, Canada } \\ \text { Smith } & \text { Norval } & \text { University of Amsterdam, Netherlands } \\ \text { Smith } & \text { Caroline } & \text { University of New Mexico, Albuquerque, Mexico } \\ \text { Thomason } & \text { Sarah G. } & \text { Michigan University, USA } \\ \text { Véronique } & \text { Georges D. } & \text { Université d'Aix-en-Provence, France } \\ \text { Winford } & \text { Donald } & \text { Ohio State University, USA } \\ \text { Wolff } & \text { Ekkehard } & \text { Leipzig University, Germany } \\ \text { Woolard } & \text { Kathryn } & \text { University of California, San Diego, USA }\end{array}$

